\title{
Determinación de la Calidad del Servicio Odontológico de un Centro de Salud Pública Basado en Intangibles. Talcahuano, Chile
}

\author{
Determination of Quality in Dental Services from a Public \\ Health Care Center Based on Intangibles. Talcahuano, Chile
}

\author{
Carlos Araya Vallespir*; Alex Bustos Leal;; Francisca Castillo*; \\ Patricio Oliva Belmar* \& Javier Araya Gozalvo***
}

\begin{abstract}
ARAYA, V. C.; BUSTOS, L. A.; CASTILLO, F.; OLIVA, B. P. \& ARAYA, G. J. Determinación de la calidad del servicio odontológico de un centro de salud pública basado en intangibles. Talcahuano. Chile. Int. J. Odontostomat., 6(3):349-354, 2012.

RESUMEN: Mejorar las actividades humanas requiere un proceso de evaluación y lo frecuente es basarse en las cosas tangibles o materiales. Sin embargo, evaluar la calidad de la atención odontológica desde la perspectiva del usuario es cada vez más frecuente, siendo la satisfacción un buen predictor. El cuestionario y su aplicación es uno de los métodos más eficaces para la detección de los niveles de satisfacción del cliente/usuario, teniendo presente el respeto a las sensibilidades y emociones que pueden dejar oculto o sobredimensionado algún indicador. El presente trabajo utilizó la escala SERVPERF, validada en empresas privadas de servicio, para determinar la calidad de atención Odontológica Pública, basada en consideraciones intangibles de los usuarios registrados en Centros de Salud Familiar de Atención Primaria Urbana. Se tomó una muestra de 405 personas de un universo poblacional de 48.746, permitiéndose un error del 5\% con un nivel de confianza del $95 \%$ y se aplicó en sus hogares una encuesta elaborada para la ocasión. La valoración más baja fue la folletería y recetarios con un 4,85 en la escala 1 a 7 , siendo este último lo mejor. Un 6,84 fue la nota asignada al sistema de acopio de datos y un 4,91 para los demás miembros del consultorio. Un 5,79 es la nota que obtienen los odontólogos que otorgan la prestación. El criterio Empatía resultó ser la media más baja y distinta a todos ( $p<0,004)$. Se puede concluir que es necesario explicar a las autoridades, que si bien los esfuerzos en equipamiento e infraestructura son útiles, debe haber un apoyo al crecimiento del capital humano ya que su valoración es un complemento importante en la satisfacción del usuario.
\end{abstract}

PALABRAS CLAVE: calidad de los servicios, intangibles de los servicios, SERVPERF, servicio público.

\section{INTRODUCCIÓN}

Las condiciones de salud de la población presentan un desajuste en la demanda respecto a los servicios que la sociedad en su conjunto ofrece, esto indica que es necesario verificar y equilibrar la situación, y que requiere una permanente evaluación del estado de aceptación o rechazo de la atención prestada. Es clásica la gran importancia a la eficiencia productiva como parte de la calidad del sistema que produce la atención (Donabedian et al., 1982).
Los intentos de mejoramiento se efectúan a nivel de macro gestión, con ajustes de costos y eficiencia, basados en las pautas del libre mercado, en donde rara vez es considerado el sentir del demandante del servicio respecto de sus necesidades, expectativas y aprensiones como "consumidor", quien al no sentir satisfacción puede tener actitudes negativas haciendo el trabajo para las personas que intervienen en el área de los servicios difícil y desmoralizante. Las 
normas de la empresa pueden ser sofocantes al impregnarse de las reglas de la competitividad y de la productividad, que pretenden dar servicio a un gran número de clientes/usuarios para mejorar la rentabilidad, con un resultado abrumador. La fatiga del final del día daría lugar a desequilibrios emocionales en quienes producen. El trabajo extra provocaría que los empleados sean menos eficientes debido al cansancio acumulado, aunque tengan la experiencia técnica necesaria para producir resultados óptimos. La descripción del "burnout" o incendio de los trabajadores de la salud fue reflejado en una investigación de Atance (1997) y se ve plenamente vigente en la atención pública hoy (Araya et al., 2011).

La definición y medición de la calidad del servicio resulta compleja, no existiendo aun consensos claros y precisos. Las escalas utilizadas para su medición entrañan un problema no superado en la literatura. A esto debe agregarse las dificultades con respecto a las características diferenciales del servicio, respecto a otras actividades sometidas a evaluación, tal como la heterogeneidad, intangibilidad e inseparabilidad (Rodriguez, 2000), consideraciones inherente a su característica de "no tangible" y que forman parte de la calidad.

No existe suficiente investigación en la búsqueda del grado de satisfacción del usuario basado en lo intangible, y menos aún en el área de la salud oral (Conde \& Alfonso, 2002; Cortés et al., 2002; Beltrán \& Trujillo, 2002; Donabedian et al.; Windström et al., 2004). Los estudios sobre reclamos por disconformidad del usuario son referidos a la eficiencia clínica; negligencias profesionales, mala praxis y falta de recursos materiales (Peña et al., 2004; González et al., 2003).

Los Intangibles. El activo intangible es creado, desarrollado y acumulado dentro de la organización por sus integrantes, con un alto y complejo componente social. A medida que la organización crece en conocimiento y habilidades, crece este activo y pensado en la optimización de los recursos, con implantación de buenos y controlados sistemas de costo (Beltrán \& Trujillo), con un mejor ambiente de trabajo para el personal del área, cada vez más afectado por el stress de su actividad, se logra un usuario más contento y satisfecho por la atención. Esto último es el superávit del Servicio Público (Bossi et al., 2001).

Evaluar la calidad de la atención desde la perspectiva del usuario y basado en elementos intangibles, es cada vez más frecuente y necesario para conocer el contexto en que se aplican las políticas públicas y valorar sus repercusiones, el grado de aceptación o rechazo de los ciudadanos a los servicios, que son de y para ellos (Arévalo et al., 1995). Es semejante a la empresa privada que se esfuerza para auscultar, atender y mantener a sus clientes completamente fidelizados.

EI SERVPERF. Un avance para la medición de la calidad de los servicios basado en intangibles, realizada mediante la discrepancia entre expectativas y percepciones de los consumidores, es el SERVQUAL desarrollado por Zeithaml et al. (1993) y consiste en un instrumento resumido de escala múltiple, de confirmada fiabilidad y validez, de 22 ítems, con una reagrupación de las dimensiones en 5 factores o criterios.

- Tangibilidad: Apariencia de las instalaciones físicas, equipos, personal y materiales de comunicación.

- Fiabilidad: Habilidad para realizar el servicio prometido de forma fiable y cuidadosa.

- Capacidad de respuesta: Disposición y voluntad para ayudar a los usuarios y proporcionar un servicio rápido.

- Seguridad: Conocimiento y atención mostrado por los empleados y sus habilidades para inspirar credibilidad y confianza. Encierra las dimensiones profesionalidad, cortesía, credibilidad y seguridad.

- Empatía: Atención individualizada que ofrecen las empresas a sus consumidores. Incorpora las dimensiones accesibilidad, comunicación, comprensión del usuario.

Las expectativas son los componentes del instrumento SERVQUAL que más controversias han suscitado por los problemas de interpretación de los encuestados, ya que las percepciones están influenciadas por las expectativas y por su variabilidad en los diferentes momentos de la prestación del servicio.

Las investigaciones realizadas por Cronin \& Taylor (1994) en 8 empresas de servicios, mediante cuestionarios, contrastaron la medición de la calidad realizada mediante la discrepancia entre expectativas y percepciones de los consumidores y la realizada solamente con las percepciones de los mismos, eliminando las primeras por ser distorsionadas por factores externos al servicio: la comunicación boca a boca, 
las necesidades personales, las experiencias pasadas y la comunicación externa de los proveedores o gestionadores del servicio. Se concluyó que una medida basada sólo en las percepciones podría ser la forma más adecuada de evaluar la calidad de servicio, surgiendo así la escala denominada SERVPERF, que presenta menores problemas de validez y es más corta, haciendo más cómoda la contestación, ya que sólo considera las percepciones de los clientes (Pakdil \& Harwood, 2005; Nowacki, 2005; Buttle, 1996).

En el presente estudio se utilizó el instrumento encuesta aleatoria bajo la norma SERVPERF, para determinar la calidad de atención odontológica pública, basada en consideraciones intangibles, de los usuarios registrados en un centro de salud familiar (CESFAM) de atención primaria urbana. Se buscó determinar el nivel general de satisfacción de los usuarios y los niveles de los criterios SERVPERF de la calidad del servicio: tangibilidad, fiabilidad, seguridad, capacidad de respuesta y empatía.

\section{MATERIAL Y MÉTODO}

Esta es una investigación observacional, transversal y descriptiva, realizada a personas atendidas en el servicio odontológico del CESFAM Paulina Avendaño, dependiente del Hospital Higueras, Servicio de Salud de Talcahuano.

Se efectúo un muestreo por sectores poblacionales, considerando como unidad la familia. Se encuestó a un usuario mayor de 14 años por hogar y habiendo obtenido previamente el consentimiento informado. El encuestador fue previamente calibrado y adiestrado en la metodología y comunicación para este proceso. Para la determinación de la muestra se realizó un estudio piloto con el objeto de obtener la varianza poblacional. De las 24912 familias asignadas al CESFAM se obtuvo una muestra final de $405 \mathrm{y}$ las encuestas se distribuyeron por sectores estratificados.

Los resultados se llevaron a una base de datos Excel y con el programa computacional Infostat se procesó la información determinando promedios y comparaciones.

Como variables independientes se consideró la edad, sexo, ocupación, nivel de estudios, sistema de atención y clasificación socio-económica.

\section{RESULTADOS}

Se verificó la confiabilidad de la escala SERVPERF, en su aplicación como instrumento de medida para la población estudiada, mediante el test estadístico Alpha de Cronbach, con un valor 0,925 para los 22 ítems de la encuesta, que la ubica como un instrumento excelente.

Se realizó estadística no paramétrica porque el comportamiento de las variables no se ajustaba a una distribución normal. El test Kruskal Wallis arrojó un $p=0,9697$ no encontrando diferencias estadísticamente significativas entre los 22 ítems señalados en la Tabla I.

En las declaraciones de acuerdo a las criterios se observa que en la visión Tangible, sin el respaldo técnico de las consideraciones tangibles del servicio, posee la más alta valoración el aspecto de la pulcritud y limpieza del consultorio con un 6.45 y valoran mal $(4,85)$ los elementos de apoyo, como folletos, instrucciones, recetas e informativos en general, desde el punto de vista si son atractivos, bonitos o llamativos. Se encontraron diferencias estadísticamente significativas entre las 4 dimensiones que constituyen este criterio (Friedman $p<$ $0,001)$.

Sobre el criterio la Fiabilidad o Confianza en el servicio recibido se encuentra la nota más alta de todos los promedios con un 6,84 y corresponde al trabajo de registro de los datos, labor netamente administrativa. Las demás respuestas están todas bajo el promedio general destacando el no respeto por las horas predeterminadas con un 5,02. Las diferencias son estadísticamente significativas (Friedman $\mathrm{p}<0,0001)$.

La Capacidad de Respuesta es el criterio que lleva en forma más pareja una tendencia sobre el promedio general, bajando en la pregunta $\mathrm{V} 13$ que dice: "los demás funcionarios del consultorio dental nunca están demasiado ocupados" con un 4,71 . Las diferencias son estadísticamente significativas (Friedman $p<0,0001$ ).

En cuanto al conocimiento que inspiran los integrantes del consultorio y si la atención otorgada le da Seguridad, el promedio se eleva sobre el general manteniendo 3 de sus valores allí y sólo uno prácticamente igualado con un 5,54. Este último valor es 
Tabla I. Promedios de las Declaraciones a los 22 Ítems SERVPERF en Pacientes Atendidos en el CESFAM Paulina Avendaño, Talcahuano.

\begin{tabular}{|c|c|c|c|c|c|}
\hline Criterios SERVPERF & Dimensiones & & Media & Media & DE \\
\hline \multirow{4}{*}{ Tangibilidad } & Equipamiento Moderno & V1 & 5,36 & \multirow{4}{*}{5,52} & \multirow{4}{*}{1,03} \\
\hline & Instalaciones Atractivas & V2 & 5,40 & & \\
\hline & Apariencia Pulcra y Limpia & V3 & 6,45 & & \\
\hline & Folletería Bonita-Atractiva & V4 & 4,85 & & \\
\hline \multirow{4}{*}{ Fiabilidad } & Respetan Horas Predeterminadas & V5 & 5,02 & \multirow{5}{*}{5,67} & \multirow{5}{*}{1,23} \\
\hline & Interés por sus Problemas & V6 & 5,48 & & \\
\hline & Tratamiento Bien $1^{\mathrm{a}}$ Vez & V7 & 5,52 & & \\
\hline & Concluyen Tiempo Programado & V8 & 5,46 & & \\
\hline \multirow{5}{*}{ Capacidad de Respuesta } & Datos Bien Registrados & V9 & 6,84 & & \\
\hline & Informan Término Atención & V10 & 5,99 & \multirow{4}{*}{5,54} & \multirow{4}{*}{1,23} \\
\hline & Atención Rápida y Efectiva & V11 & 5,80 & & \\
\hline & Demás Funcionarios Ayudan & V12 & 5,66 & & \\
\hline & Demás Funcionarios Ocupados & V13 & 4,71 & & \\
\hline \multirow{4}{*}{ Seguridad } & Da Seguridad la Atención & V14 & 5,54 & \multirow{4}{*}{5,70} & \multirow{4}{*}{1,44} \\
\hline & Dentista y Equipo Confianza & V15 & 5,76 & & \\
\hline & Son Siempre Amables & V16 & 5,80 & & \\
\hline & Conocimientos Suficientes & V17 & 5,70 & & \\
\hline \multirow{6}{*}{ Empatía } & Dentista le Saluda y le Nombra & V18 & 5,79 & \multirow{5}{*}{5,35} & \multirow{5}{*}{1,44} \\
\hline & Horarios Adecuados al Suyo & V19 & 5,55 & & \\
\hline & Le Ubican los Miembros Consultorios & V20 & 4,91 & & \\
\hline & Interés por Usted & V21 & 5,28 & & \\
\hline & Comprenden sus Necesidades & V22 & 5,22 & & \\
\hline & Promedio & & 5,55 & 1,03 & \\
\hline
\end{tabular}

el que representa al profesional en esta dimensión. El análisis estadístico con Friedman establece un $p=0,006$ indicando que las diferencias son estadísticamente significativas.

El último de los criterios que se busca detectar es la Empatía, aquella atención individualizada, con un alto componente de personalización y comprensión del usuario. La única de las 5 declaraciones que está sobre el promedio general es "el dentista le saluda y le llama por su nombre" con un 5,79. La búsqueda de un horario adecuado está en el promedio con un 5,55, pero los otros ítems como son "comprenden sus necesidades", "siente que existe un real interés por Ud." y "los miembros del consultorio le dan una atención personalizada. Le ubican" caen hasta llegar, en esta última situación, al 4,91 como promedio. Las diferencias son estadísticamente significativas (Friedman $p<0,0001)$. La media de la dimensión empatía está bajo el promedio general y alcanza a un 5,35.
En este estudio prevalece la opinión de las personas de sexo femenino, sobre las de sexo masculino, por dos razones: haber sido efectuada en los hogares en horario donde el proveedor estaba trabajando y por la costumbre de ser la mujer quien frecuentemente se relaciona con los problemas de salud de la familia.

El número de actividades laborales de los proveedores del hogar es variada y mayoritariamente ocupada (sólo un $6 \%$ de cesantía declarada) lo cual quita una sensación de mayor resentimiento en las respuestas. Los niveles de educación corresponden a una población de nivel medio, no encontrándose entre los encuestados personas analfabetas.

Los niveles socioeconómicos basados en el indicador de la previsión que presentaban los usuarios hablan de un predominio de clase media y media baja. Son los principales beneficiarios de los sistemas públicos de salud. 


\section{DISCUSIÓN}

El promedio general de 5,5 para todos las dimensiones coincide con trabajos similares donde la aprobación se ubica siempre entre el 70 y el $80 \%$ (Ramirez et al., 1998; Rey, 2000; Lara et al., 2000; Caminal, 2001; Jiménez et al., 2002; Sosa et al., 2005) y no significa una deficiente evaluación del servicio prestado por CESFAM, más aún si se observa que uno de los puntos bajos "la folletería" no es difícil de remontar. Sin embargo, no serán fáciles los correspondientes al personal de apoyo y el trato al usuario, revertir la situación requiere esfuerzos para mejorar el capital humano o una mejor distribución de la fuerza laboral para incrementar la eficiencia del servicio (Bossi et al.) sin que implique el deterioro de otras áreas bien evaluadas (Cortés et al.; Lorente et al., 2001) que es necesario destacar, como son el manejo de la información y la apariencia del consultorio con excelentes notas: 6,84 y 6,45 respectivamente.

Otro de los temas conflictivos de la atención en salud es el respeto por el tiempo de los usuarios (Ramírez et al.; Jiménez et al.; Retamal \& Monge, 2000). El cumplimiento en los horarios de citas y el plan de término de los tratamientos es otra tarea pendiente en esta área.

Al ser real la situación de disponibilidad de poco tiempo del funcionario del CESFAM es de cuidado el riesgo del Burnout (Atance; Araya et al.).

De los criterios que detectan la empatía las valoraciones se pueden potenciar, con una mejora sustantiva en lo que es saludar y conocer de nombre a los usuarios y sus familiares en el proceso de atención. Engloba esta percepción la mejora en la comunicación entre el profesional, su equipo, el usuario y su entorno (González et al.).

De los cinco criterios considerados la Empatía presenta el más bajo con un $5,35(p<0,004)$. Se podría decir que se hace el trabajo con un: "falta ponerse en el lugar del otro."

El impacto que pueda tener el reconocimiento de las falencias detectadas en el presente trabajo por parte de las autoridades sanitarias y los profesionales que trabajan en los servicios de salud, debe generar una mejoría en las condicionantes de la calidad de la atención a la comunidad beneficiaria. Creará, necesariamente, un actuar más participativo e integrado en el proyecto conjunto de la mantención de la salud individual, familiar y de la sociedad.

ARAYA, V. C.; BUSTOS, L. A.; CASTILLO, F.; OLIVA, B. P. \& ARAYA, G. J. Determination of quality in dental services from a public health care center based on intangibles. Talcahuano, Chile. Int. J. Odontostomat., 6(3):349-354, 2012.

ABSTRACT: Improving human activities requires an evaluation process, in more cases based on tangible or material things. However, assessing the quality of dental care from users perspective is becoming more common, using a satisfaction concept as a good predictor. The questionnaire and its implementation is one of the most effective methods for evaluating customer/users satisfaction levels, bearing in mind the respect for sensitivities and emotions that can leave some hidden or oversized answers. This study was design to determine the quality of public dental care service, using a private service validated scale "SERVPERF", that is based on intangible considerations of registered users from Family Health Centers of Urban Primary Health Care. A sample of 405 people from a population of 48,746 was taken, $5 \%$ error and $95 \%$ of confidence level was allowed and a specially prepared survey was applied at home. The lowest rating obtained was 4.85 for the brochures and recipes on a scale from 1 to 7 , being the last one the best. A note 6.84 was assigned to the data collection system and a 4.91 for the other members of the service. For dentist who provides the service, the score was 5.79 . The empathy criterion proved to be the smallest average obtained from all others concepts $(p<0.004)$. We conclude that it is necessary to explain to the authorities the utility of equipment and infrastructure, and there must be a support to the growth of human capital as an important complement for the users satisfaction.

KEY WORDS: quality of services, intangible services, SERVPERF, public service.

\section{REFERENCIAS BIBLIOGRÁFICAS}

Araya, C.; Oliva, P.; Ananías, N.; De Los Santos, P. \& Mendoza, M. Trastornos ansiosos y desórdenes temporomandibulares en funcionarios de un centro de salud familiar en la comuna de Concepción. Chile. Int. J. Odontostomat., 5(3):235-9, 2011.

Arévalo, R.; Polo, P. \& Sivit, C. Políticas públicas y estudios de opinión, una colaboración con futuro. Rev. Esp. Salud Pública, 69(3-4):259-63, 1995. 
Atance, J. Aspectos epidemiológicos del síndrome de Burnout en personal sanitario. Rev. Esp. Salud Pública, 71(3):293-303, 1997.

Beltrán, J. \& Trujillo, M. Perfeccionamiento Institucional en el sector salud. Proyección del director. Rev. Cubana Salud Pública, 28(1):5-17, 2002.

Bossi, A.; Fuertes, Y. \& Serrano, C. El Capital Intelectual en el Sector Público, 2001. Disponible en: http:// www.5campus.com/leccion/cipub

Buttle, F. SERVQUAL: review, critique, research agenda. Eur. J. Market., 30(1):8-32, 1996.

Caminal, J. La medida de la satisfacción: un instrumento de participación de la población en la mejora de la calidad de los servicio sanitarios. Rev. Calidad Asistencial, 16:276-9, 2001.

Cortés, A.; Flor, E. \& Duque, G. Análisis de costos de la atención médica hospitalaria. Experiencia en una clínica privada de nivel II-III. Colomb. Med., 33:45-51, 2002.

Conde, J. \& Alfonso, L. Propuesta metodológica para evaluar un servicio estomatológico. Rev. Cubana Estomatol., 40(1):37-45, 2002.

Cronin J. J. \& Taylor, S. A. SERVPERF versus SERQUAL: Reconciling performance-based and perceptions- minusexpectations measurement of service quality. J. Market., 1994; 58(1):125-31, 1994.

Donabedian, A.; Wheeler, J. \& Wyszewianski, L. Calidad, Costo y Salud: Un modelo integrador. Medical Care, 20(10):975- 92, 1982.

González, S.; Jiménez, M. E.; Triana, J.; Ureña, J. L.; García, J.; Carrillo, J.; Rivera, A. E.; Manuel, G.; Sánchez, J. M. \& Tena, C. Recomendaciones para mejorar la práctica Odontológica. Rev. Conamed., 8(1):29-38, 2003.

Jiménez, M.; Ortega, M.; Cruz, M.; Quintero, M.; Mendoza, E.; Antúnez, M.; Solís, M. \& Hernández, M. Satisfacción del usuario como indicador de calidad. Rev. Mex. Enferm. Cardiol., 11(2):58-65, 2002.

Lara, N.; López, V.; Morales, S. \& Cortés, E. Satisfacción del usuario de dos servicios odontológicos en Tláhuac, Distrito federal. Rev. ADM, LVII(5):175-9, 2000.

Lorente, J.; Velásquez, A. \& Núñez, A. Algunas reflexiones sobre la calidad en la prestación de los servicios de salud. Medisan, 5(2):34-7, 2001.

Nowacki, M. Evaluating a museum as a tourist product using the SERVPERF method. Museum Management and Curatorship, 26:1-16, 2005.
Pakdil, F. \& Harwood, T. Patient satisfaction in a preoperative assessment clinic: An analysis using SERVQUAL dimensions. Total Qual. Manag. Bus. Excel., 16(1):1530, 2005.

Peña, R.; Rodríguez, J. R. \& López, J. M. Factores asociados a la gestión de quejas contra médicos que laboran en hospitales del Instituto Mexicano del Seguro Social. Salud Pública Méx., 46:210-5, 2004.

Ramírez, T.; Nájera, P. \& Nigenda, G. Percepción de la calidad de la atención de los servicios de salud en México: perspectiva de los usuarios. Salud Pública Mex., 40:312, 1998.

Retamal, A. \& Monge, V. Calidad percibida por dos poblaciones adscritas a dos centros de salud de la provincia de Cuenca. Rev. Esp. Salud Publica, 74:275-86, 2000.

Rey, C. La satisfacción del usuario: un concepto en alza. Anales de Documentación, 3:139-53, 2000.

Rodríguez, O. A. Calidad en los servicios de salud en Colombia desde la perspectiva del consumidor: propuesta de construcción de una escala de medida. Rev. Colomb. Market., 1(1):1-12, 2000. Disponible en: http:// revistas.unab.edu.co/index.php?journal=marketing \& page $=$ article $\&$ op $=$ view $\&$ path []$=783 \&$ path []$=760$

Sosa, G. B.; Sosa, C. A. G. \& Gómez, J. J. Niveles de satisfacción y condicionantes de la consulta de los usuarios en los servicios de guardia hospitalaria. Universidad Nacional del Nordeste, Argentina, 2005. Disponible en: http://www.unne.edu.ar/unnevieja/Web/cyt/cyt/sociales/ s-012.pdf.

Widström, E.; Linna, M. \& Niskanen, T. Productive efficiency and its determinants in the Finnish Public Dental Service. Community Dent. Oral Epidemiol., 32(1):31-40, 2004.

Zeithaml, V.; Parasuraman, A. \& Berry, L. Calidad total en la gestión de servicios. Madrid, Díaz de Santos, 1993. pp.17-54.

Dirección para Correspondencia:

Dr. Carlos Araya Vallespir

Facultad de Odontología

Universidad de Concepción

Avenida Roosevelt 1550

Concepción

CHILE

Email: carlos.araya@udec.cl

Recibido : 30-04-2012

Aceptado: 27-08-2012 